\title{
Book Abstract: How to Write an Emergency Plan by David Alexander; Reproduced by Permission
}

\author{
David Alexander ${ }^{1}$
}

1. UCL Institute for Risk and Disaster Reduction, University College London, London, UK.

Crtation: Alexander D. Book Abstract: How to Write an Emergency Plan by David Alexander; Reproduced by Permission. Health in Emergencies and Disasters Quarterly. 2016; 1(4):215-224. https://doi.org/10.18869/nrip.hdq.1.4.215

: https://doi.org/10.18869/nrip.hdq.1.4.215

Article info:

Received: 18 Jan. 2016

Accepted: 28 May 2016

\section{Keywords:}

Emergency Planning, Disaster, Emergency management

\section{A BSTRACT}

In 2002, David Alexander wrote Principles of Emergency Planning and Management. Long a standard reference work in that book he described the processes of preparing for and responding to disasters, crises, and civil contingencies. He based the work on principles because he wanted to dissociate it from any particular system of emergency management. Then, as now, many books in this subject are tied to individual systems of public administration, particularly the federal system of the United States of America, which somewhat limits their usefulness in other contexts. However, there is no 'one-size-fits-all' civil protection system that can act as a template for all eventualities. Nevertheless, David Alexander does not believe that, in terms of how emergency response is organized, there must necessarily be fundamental differences between the world's richest and poorest countries. They all need safety and rapid response to civil contingencies. All countries can learn from others and derive benefit from incorporating good practice from abroad into their own systems, with modifications to fit local conditions. Equipment may be expensive, but planning to make the best use of what one has or can afford is not likely to break the bank.

The feedback he received from the Principles book was generally positive, but it indicated that some readers were facing with severe practical problems in frontline jobs that required them to provide workable answers. Many were new to emergency planning and did not know where to start. So, when David Alexander found the time to consider building on his earlier Principles, he decided to give it a more 'hands on' profile and to endeavor in meeting the needs of people who had been given the task of writing and implementing emergency plans. To maximize the geographical scope of the book, he decided to stay with his decision not to tie the explanations to particular systems and countries, in the expectation that users of the new book will be able to make the necessary connections and adapt his general approaches to the configuration of services in any country or region.

Some readers may be skeptical about the value of emergency planning. It is true that in a crisis the first thing that goes out of the window is the plan. However, David Alexander believes passionately, not in the plan as a document or instrument, but in the process of planning. He believes that emergency planning should be a flexible process that is able to adapt to dynamically changing circumstances. Moreover, the process must not stop when a basic document, 'the plan,' has been prepared: it should continue right through the next emergency to the recovery phases.

An emergency plan is a structured document, an instrument that outlines the responses envisaged for certain kinds of civil contingency, such as a flood, a major snowstorm or a transportation crash with casualties. Emergency planning is simultaneously an art and a science. It involves 'thinking the unthinkable', so that people can be ready for those aspects of an emergency that require preparation beforehand because they cannot effectively be improvised during the crisis. There are so many ramifications of emergency planning that not all of them can be covered in a book such as "How to Write an Emergency Plan." However, the skill that must be learned is to think through the implications of prior decision-making. Some implications are obvious but many are not, which is one of the main justifications for writing this book. There are good emergency plans and there are bad ones. A plan may be too obtuse, complex, grandiose, idealistic; too detailed or too general; or good in theory but bad in practice. Thus there is always room to improve. The failure of a plan to solve the problem of how to respond to an emergency does not invalidate planning as such; rather, it demands a better plan and a renewed effort to improve the process of planning.

Emergency planning is a social endeavor that needs to be backed by hard scientific information; for example, on the magnitude and frequency of certain hazard impacts. By 'social' David Alexander means that it should involve consultation and collaboration. Often, emergency planning is as much a political 
process as a technical one. Public administration, hazard and risk science, journalism, technical response, welfare and other services must work in concert. The emergency plan is their script, and as such it is something with which they all need to feel comfortable and familiar. This book should convince readers of the importance of good emergency planning, that it is intended to disseminate a methodology and also provide a justification for more and better emergency plans.

Nowhere on earth is free from hazard and risk. Hence, emergency planning is not a luxury, but a necessity. In writing this new practical book, David Alexander has not abandoned the issue of principles. So the user will find these stated at intervals in the text. His intention is to provide some guiding markers along the route to explain the emergency planning process. His aim overall is to 'demystify' a process that, in many respects, is a form of 'codified common sense', but one in which the challenge is to do much more than merely thinking sensibly. The issues, and the connections between them, are complex; hence the need for a book that sets them down in print, in some kind of logical order, or what Albert Einstein described as 'a feeling for the order lying behind the experience.'

Contents: Foreword. 1. Introduction. Scope and objectives of this book; 2. What are emergencies? 3. What is an emergency plan? 4. The emergency planning process; 5. First step: background research; 6. Second step: scenario building; 7. Third Step: from scenarios to actions; 8 . A note on the structure of the plan; 9. Fourth step: using the plan; 10. Planning to maintain the continuity of normal activities; 11. Specialized emergency planning; 12. Conclusion: the future of emergency planning. Afterword. Appendix 1: Glossary of working definitions by key terms. Appendix 2: Bibliography of selected references. Index.

\section{Scope and Objectives of This Book}

mergency plans are becoming increasingly important in modern life. They reflect the gradual realization during the 21 st century that there is no justifica tion for failure to prepare for adverse events whose occurrence can be foreseen or predicted. They also reflect a potentially more hazardous world af- fected by the gathering pace of global change, a world whose sheer complexity makes it more vulnerable to threats and hazards. The alternative to making plans that help one tackle crises logically and rationally is improvisation. All emergencies have some uniqueness, which cannot be ruled out completely, and if it gets the upper hand the result is almost always inefficiency. In emergency response this usually manifests in casualties that can be avoided and damages that can be reduced.

Emergency plans are needed, and perhaps legally required, for a wide variety of civil institutions, including the following:

- Public administrations at the local, regional, and national levels,

- International bodies such as the United Nations and European Union,

- Factories that produce hazardous substances or use dangerous production processes,

- Businesses that could suffer severe disruption or damage as a result of adverse events,
- Key facilities, such as airports and hospitals, and other forms of critical infrastructures, and

- Cultural heritage sites such as museums, galleries, archives, and archaeological sites.

Principle: Emergency planning is an important activity for many organizations and different areas of jurisdiction

The processes of writing, disseminating, utilizing, and maintaining plans are becoming increasingly well known, and the whole emergency management field is gradually turning into a fully-fledged profession. However, it is by no means uncommon to find that plans are sub-standard, i.e., they have little chance of working effectively during a crisis situation. It is still common to encounter the 'paper plan syndrome', or its digital equivalent, in which a docu- ment is written (or a digital file is produced), which is left in a desk drawer and forgotten until something serious happens. At that point it is too late to learn how to use the plan.

The dead hand of an unrevised plan, with which no one is familiar, can be a serious handicap to good emergency management, for it could easily be more misleading than informative. In fact, emergency plans that are poorly structured, badly maintained or out of date can potentially be lethal. If rules, norms, or laws require plans to be made, they should be constructed in order to honor the spirit, not merely the letter of the regulations, and thus contribute positively to public safety. Hence, let us ensure that emergency plans are active, living documents, the valued possessions of their users and beneficiaries. 
Principle: An emergency plan should be a 'living document' that is kept up to date and is familiar to its users

The purpose of this book is to provide a brief, general introduction and guide to emergency planning. The aim is to demystify the processes involved and offer a structure that can be followed in order to formulate a plan and ensure that it works properly and remains functional over time. The approach is a generic one, partly because what is needed is a flexible instrument capable of producing sat-isfactory results in the case of both known, anticipated hazards (such as the inundation of a river floodplain) and unexpected ones (such as a major transportation crash with an environmental release of toxic substances).

The use of a generic approach means that the book does not follow any one civil protection or emergency preparedness system. Instead, it relies on general principles established by more than half a century of intensive worldwide research on crises and disas- ters. The reader should be able to adapt the principles described to any particular national or local system of emergency management.

Emergency planning is a continuous process. We live in a world that is constantly and rapidly changing. The changes are reflected in adjustments at the local and global levels and emergency plans must take account of them. The science and art of constructing a plan involve many complex issues of a multi-disciplinary nature.

Principle: Emergency planning is a multidisciplinary process that cuts across the boundaries of professions and disciplines

Hence, this book offers an approach that is perhaps more comprehensive than what most emergency planners will need. This enables one to consider a wide range of situations and exigencies. The reader is urged to be selective in what he or she uses. An emergency plan should aim to be comprehensive in what it seeks to do, but elegant in how it sets out its provisions. In other words, it is important to create an instrument in which one can see the wood for the trees.

Principle: Emergency planning should strike a balance between providing detail and ensuring that its structure is clear to the users

This book cannot cover all elements of the emergency planning process, which is too vast and variegated for that to be possible. Society is complex and so are its needs. The important thing here is to inculcate the skills of thinking in the right way rather than trying to discuss every possible detail of the process.

The reader who becomes skilled in emergency planning will know how to anticipate the unexpected, identify unusual linkages, and prepare for events that are highly out of the ordinary. Much of emergency planning is little more than carefully codified foresight; the rest is about developing the mental flexibility to cope with extraordinary events. That is both a science and an art.

\section{Why Write an Emergency Plan?}

No place on earth is free from the risk of disaster. The incidence of extreme events varies considerably from one place to another and over time, but there is a global trend towards more and bigger disasters, which tend to involve more people either directly as victims or indirectly as those who suffer disruption and taxation to pay for the consequences of adverse events. Around the world in an average year -if there is such a thing- there may be as many as 700 disasters, of which about $60 \%$ stem from events in nature and the rest are the result of human action or technological breakdown. The statistics, by the way, are heavily dependent on how one defines 'disaster', both in terms of the size of the event and its statistics, and whether or not one includes conflicts and epidemics. Moreover, the toll of disasters is highly variable from one year to another; hence, we have both 'good' and 'bad' years, with fewer or more disasters, or smaller or bigger ones, respectively.

Disasters can be classified into five categories, as follows:

- Natural hazards and disasters. They vary from sudden, unannounced events such as earthquakes to long-drawnout 'creeping' disasters such as drought and desertification. Floods, hurricanes or other major storms, landslides and earthquakes are the most common ones and events such as droughts, volcanic eruptions and environmental fires are also common in certain parts of the world,

- Technological disasters. Examples include transportation crashes, episodes of chronic or catastrophic pollution, oil refinery fires, and sudden loss of basic services such as electricity supply,

- Social contingencies. These include riots, demonstrations, and mass gatherings with crowd crushes. It is possible to include disease epidemics in this category, as many of their effects are not medical but socioeconomic, such as loss of personnel and absenteeism from work. Not all social contingencies are negative events, as mass gatherings may be celebrations, but 
in order to avoid calamity, these events require the same planning provisions as more disastrous events,

- Intentional disasters. They vary from conventional terrorism, including bombings and hostage taking to chemical, biological, nuclear, and radiological incidents. The strategic, planned nature of terrorist activity distinguishes this category from other forms of technological disasters, which are generally the result of human error or equipment failure.

- Hybrid, compound, and cascading events. This class includes the so-called 'natech' events, which are distinguished by the interaction of extreme natural events, such as earthquakes and floods, and technological failures, for example, computer failure or structural collapse leading to environmental pollution.

Commonly, emergencies and disasters are recurrent events. Hence, we tend to use a cyclical model to describe the process of dealing with them. Of course, not all major incidents are cyclical or even recurrent. A dam burst leading to catastrophic outburst flooding is unlikely to recur, as the dam will either be rebuilt to safer standards or not rebuilt at all. On account of seasonal effects, events with a meteorological origin are those that are most likely to be cyclical, as with, for example, monsoon flooding in the tropics, or the El Niño-Southern Oscillation in the Pacific basin and beyond. Despite its limitations, the cyclical model is attractive because it emphasizes the constant need to keep planning and preparedness up to date.

The stages of the 'disaster cycle' (Figure 1) are as follows:

- Mitigation: During times of quiescence, attention should be devoted to the management of risks and preparation for future adverse events,

- Preparation: During the lead-up time to a crisis, emergency or disaster,

- Attention will mainly be focused on preparing to tackle the event and implementing ways of reducing the impact. In some cases, where warning and lead time are sufficient, these may include evacuation of the general population,

- Emergency intervention: This is the critical period for the emergency plan, in which organizations must work together to rescue people, provide aid and assistance, and restore safe conditions,
- Recovery: The end of the emergency phase will be marked by stand-down of search and rescue (SAR) personnel and demobilization of other first-response units. At this point, restoration of basic services becomes the prime requirement. The emergency plan should ensure that vigilance prevails, as injuries and damage can occur as a result of the abnormal conditions that commonly persist after a disaster, and in case the hazard returns,

- Reconstruction: Recovery from a major disaster may take years or decades. While the reconstruction process is not directly part of the emergency plan, it furnishes an opportunity to seek greater safety and improve the integration of emergency response with other civil society functions.

Being a simplification of reality, there are many cases in which the 'disaster cycle' is neater than reality. For example, well-organized societies with plenty of resources may start the recovery and reconstruction process even before the end of the emergency period. Hence, the phases of the cycle may overlap.

Principle: Although emergency planning focuses on what to do during the emergency period of a disaster or crisis, it has a role to play in all other phases

Resilience is another useful concept, and one that has gradually become more and more fashionable among counter-disaster planners. As with so many terms in this field, there are many possible definitions, which are a product of the long and complex history of the term. One of the most useful definitions stems from work on the physics of materials. In response to an applied force, a resilient material has both the strength to resist, thus preserving its form, and the ductility to absorb stresses by deforming without breaking. By analogy, society must have robustness (strength) and adaptability (ductility) when faced with the threat and impact of disaster. As it includes limiting damage and anticipating urgent needs during a crisis, emergency planning is part of the process of creating resilience in society.

The reasons and justifications for writing an emergency plan can be summarized as follows:

- It may be a legal or procedural requirement of an organization to have such an instrument,

- By making emergency intervention more efficient and effective, a plan can help reduce damage and 


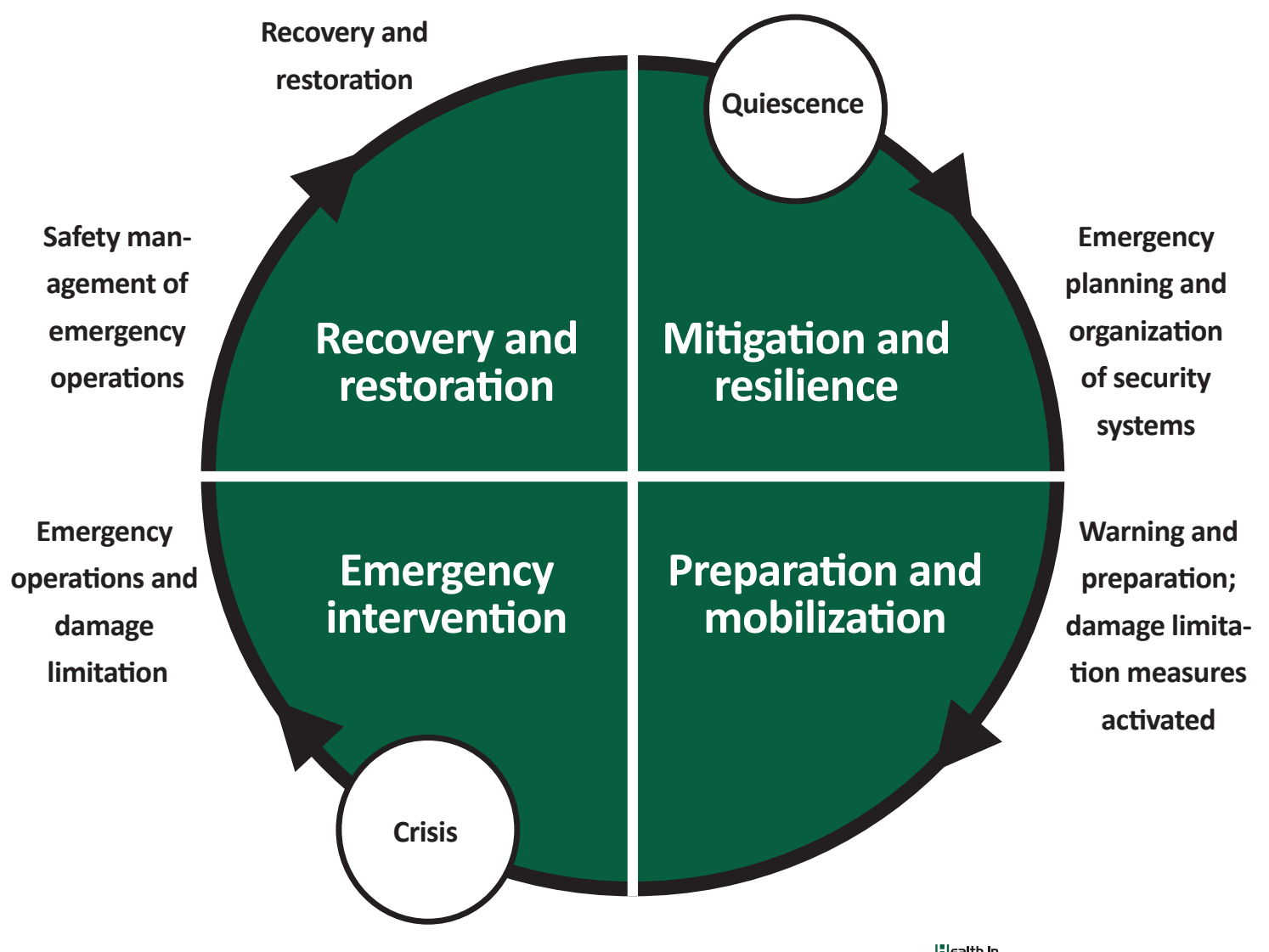

Figure 1. The 'disaster cycle' and some of the principal tasks associated with each phase.

Emergencies and |D]isasters [O]uarterly

casualties and speed up the process of restoring normal conditions,

- By foreseeing needs and apportioning tasks, plans help organizations work together,

- Emergency plans help one to understand what kinds of risk are present in an area and what needs to be done to encounter them,

- In emergency response, improvisation is a sign of inefficiency. Where it is avoidable it cannot be tolerated because the human consequences are too serious,

- Finally, planning helps simplify complex reality and make it intelligible so that it can be managed effectively.

In an emergency situation responders need to understand, not merely what their own roles are, but also what the roles of other participants are. This enables them to interact effectively, and points the way to the need for a common language and culture. Hence, managing an emergency is obviously far more than simply having a plan and following its provisions. However, a plan can help facilitate interoperability. It can do so by:
- Apportioning tasks and responsibilities,

- Specifying the conditions under which particular roles are assumed and actions are carried out,

- Providing a structure for mutual aid and complementary actions,

- Ensuring that in a crisis all necessary tasks will be carried out and all needs addressed,

- Foreseeing all predictable needs and preparing to fulfil them, and

- Helping to ensure compatibility between response units of procedures, equipment, and communications protocols.

Principle: Emergency planning is about helping to create common objectives, language and culture, for the organizations and people who respond to emergencies

Detractors have suggested that emergency plans hamper the response to a crisis because they impose constraints on processes that can be better carried out by improvis- 


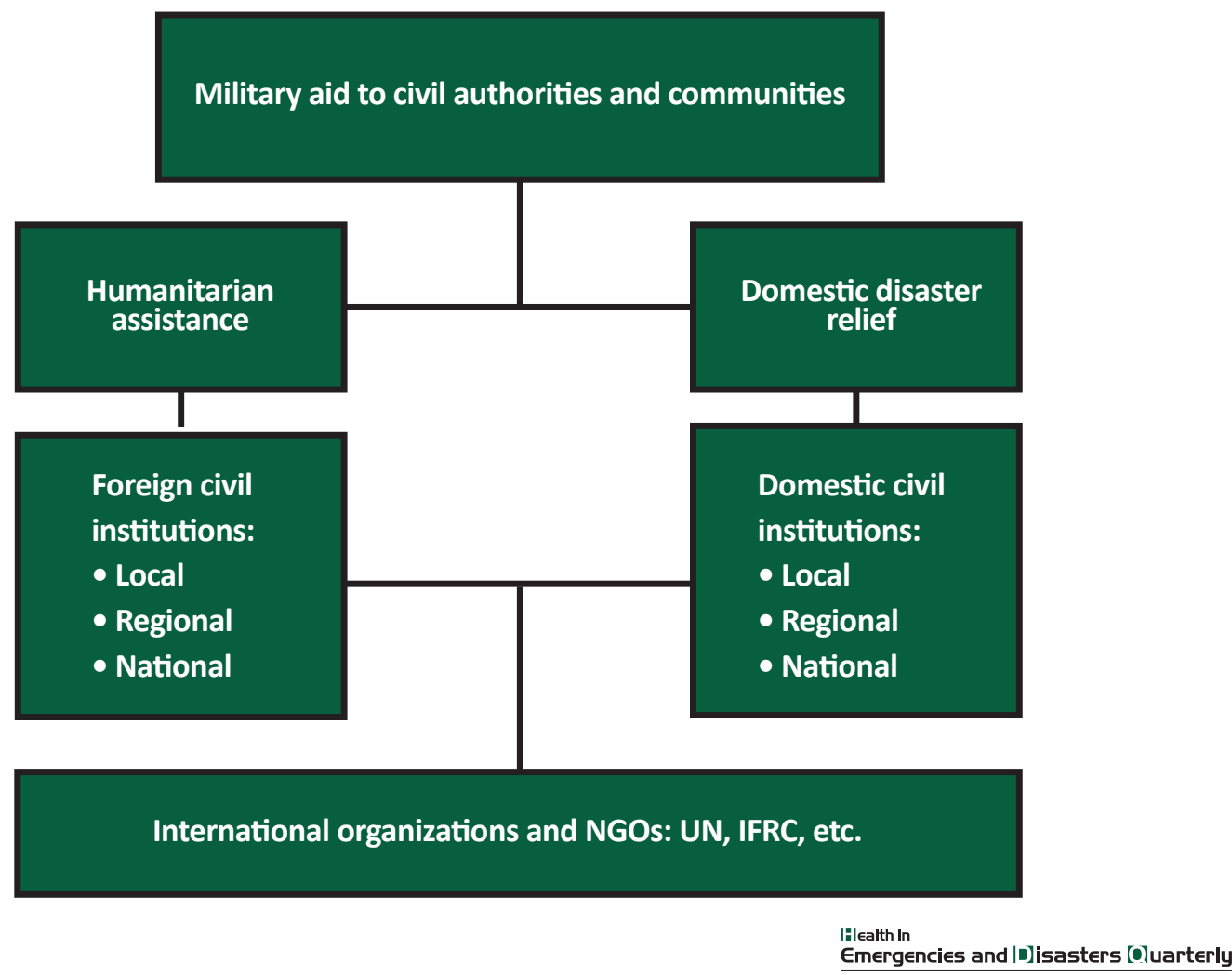

Figure 2. Summary of the role of military assistance to civilian authorities and communities in civil and humanitarian emergencies and disasters.

ing. Improvisation, they say, is more flexible than following set procedures, and hence is more sensitive to variations in the needs of the moment. There is some truth in this, but it is quite a dangerous attitude. In the first place, failure to foresee and prepare for predictable needs is tantamount to negligence.

Secondly, unless it is constrained to a minimum, improvisation is highly inefficient. During an emergency, there is unlikely to be sufficient time to work out complex procedures and protocols. Thirdly, emergency planning should be treated as an exploratory process which, if properly pursued, will reveal the complexities and intricacies of crisis situations and thus allow them to be anticipated and managed properly.

The upshot of these considerations is that emergency planning should always be treated as a flexible exercise that facilitates, rather than inhibits, the processes of getting to know a crisis and preparing to deal with it.

\section{Emergency Panning and Civil Protection Systems}

One of the main objectives of emergency planning is to coordinate the work of different forces that may either be unused to working together, or not familiar with doing so under the unusual conditions of a major crisis. Each country has its own ideas, traditions, and procedures for organizing the relevant forces: police, fire and rescue, ambulance and hospital, and volunteer civil protection organizations.

In this respect, the form of the emergency plan will depend considerably on the nature of the command systems with which it has to deal. The most complex situations occur where these differ substantially between services. However, most countries will have a common operating system of some kind. It will be based somewhere along a continuum that extends from the command principle to the support function principle (see Figure 4, below).

The former involves command and control, as derived from military experience, in which chains of command are present and a hierarchy of ranks determines the ability of participants in an emergency situation to give or receive and act upon orders.

Under the support function principle there is effectively no chain of command and all actions are the result of collaboration and co-operation. In real cases, it is unlikely that a system will fall at either end of the spectrum, but it will instead have elements of both command and collaboration. 
The tendency in the modern world is to move away from the command-and-control military model towards a more collaborative approach (even military organizations are, to some extent, doing this).

The change is encouraged by the fact that advances in information and communications technology are tending to flatten the chain of command, if such exists, by making at least some of the hierarchy redundant.

Principle: Information technology increases the opportunities for collaboration and reduces the need for a hierarchy. It therefore tends to flatten the chain of command

As an aside, it should be noted that many countries have signed a protocol on the use of military assistance in civilian disasters. This is termed the 'Oslo Guidelines on the Use of Foreign Military and Civil Defence Assets in Disaster Relief [1]. The guidelines stress the need to separate the military and humanitarian roles of armed forces. Paragraph 37 states unequivocally that military humanitarian assistance should be co-ordinated by civilian forces in the field and should respect principles of impartiality, neutrality, and sovereignty. The Oslo Guidelines refer specifically to the use of foreign forces. The domestic role of the military in civilian disasters varies considerably from one country to another. In the United Kingdom, the armed forces are frequently used in a support role. Their services are given for free if lives are at risk, but they levy charges if that is not the case. Figure 2 summarizes the options for military assistance to civil authorities and civilian communities in disaster on either a domestic or a foreign level.

Two fundamentally different modes of organization are associated with the spectrum. Command and control involves three or four basic levels of decision-making and action. In the United Kingdom, these are known as platinum, gold, silver, and bronze. Platinum is the highest echelon. It involves officials and politicians up to prime ministerial level. In Britain they meet in the Cabinet Office Briefing Room (COBR). Their task is to ensure that a crisis is being properly directed and responded to, and that all government departments are engaged as needed. This is the policy level. Gold is the strategic level, which must find resources and ensure that emergency activities are broadly going in the right direction.

The gold command headquarters may be located at some distance from emergency response activities. Silver is the tactical level, and it may occupy an emergency operations center that is either in the affected area or near to it. Tactical work involves allocating resources and coordinating individual initiatives in the field. Finally, the bronze level is operational and is co-ordinated from the emergency site. Here, resources acquired by the gold level and allocated by silver will be put to use.

The alternative model is to divide up activities by sector. The following is a list of possible sectors. It should be borne in mind that most systems will not have all of these sectors (there are 20, and the most usual number is between 10 and 16), as some may be amalgamated with others, or simply not utilized.

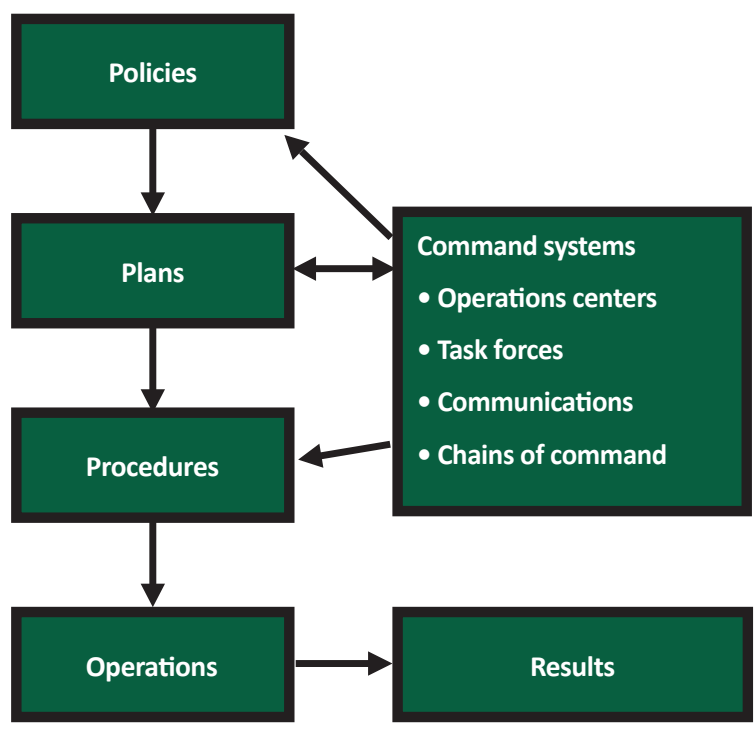

Figure 3. A 'command function' model of organization for civil protection and emergency planning. 


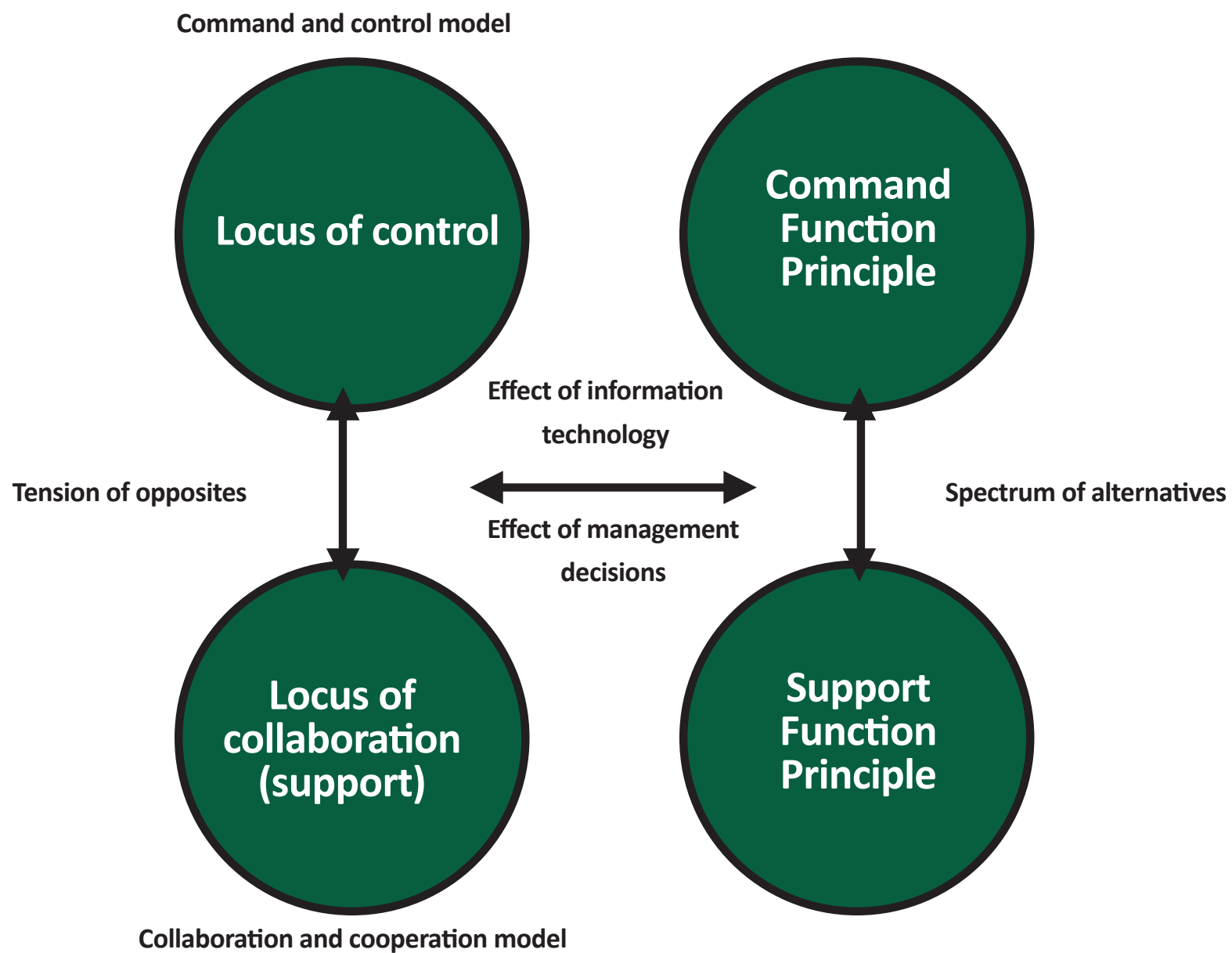

Health in

Emergencies and D]isasters [Oluarterly

Figure 4. Comparison of the 'command function' and 'support function' models of emergency management.

- Scientific, technical and planning, early warning,

- Co-ordination of emergency operations and EOCs,

- Telecommunications,

- Search and rescue,

- Evacuation logistics, recovery areas, rest centers,

- Health response, social assistance, veterinary services,

- Water, sanitation, hygiene,

- Safety, risk assessment, protection,

- Census of damage, people, things,

- Mass media, public information,

- Voluntary services,
- Materials, vehicles, support logistics,

- Transportation, traffic, access, cordoning,

- Utilities, essential services,

- Local authority liaison and support,

- Hazardous materials,

- Environmental protection,

- Nutrition, food security,

- Schools, education, and

- Finance, accountability.

The sector model is also known in the form of a 'cluster' system in which 'clusters' of organizations, or their particular representatives or task forces, work together on assignments associated with each sector. Where the support 
function principle dominates, emergency managers tend to be resource managers rather than commanders.

The alternative to a support function model is one based on command functions (Figure 3). This is more hierarchical and uses the PESTOR system described in chapter 3 . In reality, the distinction between a vertical, 'top-down' and a horizontal, collaborative model is more of a spectrum than a binary choice (Figure 4). Although there is a certain 'tension of opposites' between the two end members, there are many combinations of ingredients that can place the system at a particular point on the spectrum. Information technology facilitates sharing information, and hence promotes collaboration over command. However, whether more or less emphasis should be placed on command and control, depends much on the culture of civil protection.

Wherever a national system of civil protection falls on the continuum between command function and support function principles of organization, it is likely to have a lead agency. This will either be deliberately designated, and hence will be explicit, or will happen by default and thus be implicit. In some cases the lead agency co-ordinates the others: for example, in the United Kingdom, the police usually co-ordinate emergency operations, unless there is a pressing reason for the fire and rescue or health services to take the lead. In countries where mass casualties are greatly feared -for example, as a result of large earthquakes- the lead agency may be medical, such as the national Red Cross or Red Crescent So- ciety. Alternatively, where the biggest problem is likely to be structural collapse, the lead agency may be the fire service, which is skilful in search and rescue, as in a major disaster the other services will not be able to work until the fire service has stabilized the scene.
In modern civil emergencies, no matter how large the disaster or crisis, the theatre of operations is always local. Hence, the local response is paramount. An exception can sometimes be made for the response to terrorist outrages, for which national co-ordination may predominate, but for natural, technological and social disasters, local capability and autonomy are essential. Responses provided at the national level and organized by the intermediate tier of government (region, province, department, prefecture, county, or state) should aim to support local efforts. There is thus a need to provide a common operating picture and an integrated system in which emergency operations centers (EOCs) are linked in a hierarchy, from the grand co-ordination center at the headquar- ters of national civil protection, through the regions to the local level. In a large city, the municipal headquarters will co-ordinate the work of more local EOCs in boroughs or districts of the city.

Principle: Higher levels of government should co-ordinate and harmonize the role of local civil protection authorities

Even in the countries with the most standardized, highly articulated systems of this kind, civil protection is usually a mosaic of constituent parts, represented by varying degrees of development. This is because much depends on individual foresight, leadership and entrepreneurial spirit. Local priorities can differ considerably between neighboring jurisdictions. The law may require each unit of public administration to have plans and prepare for emergencies, but there is a difference between taking the matter to heart, in other words honoring the spirit of the law, and merely honoring the letter, or in other words doing the minimum. As a result, the performance of the

Table 1. Size of event in relation to capacity to respond.

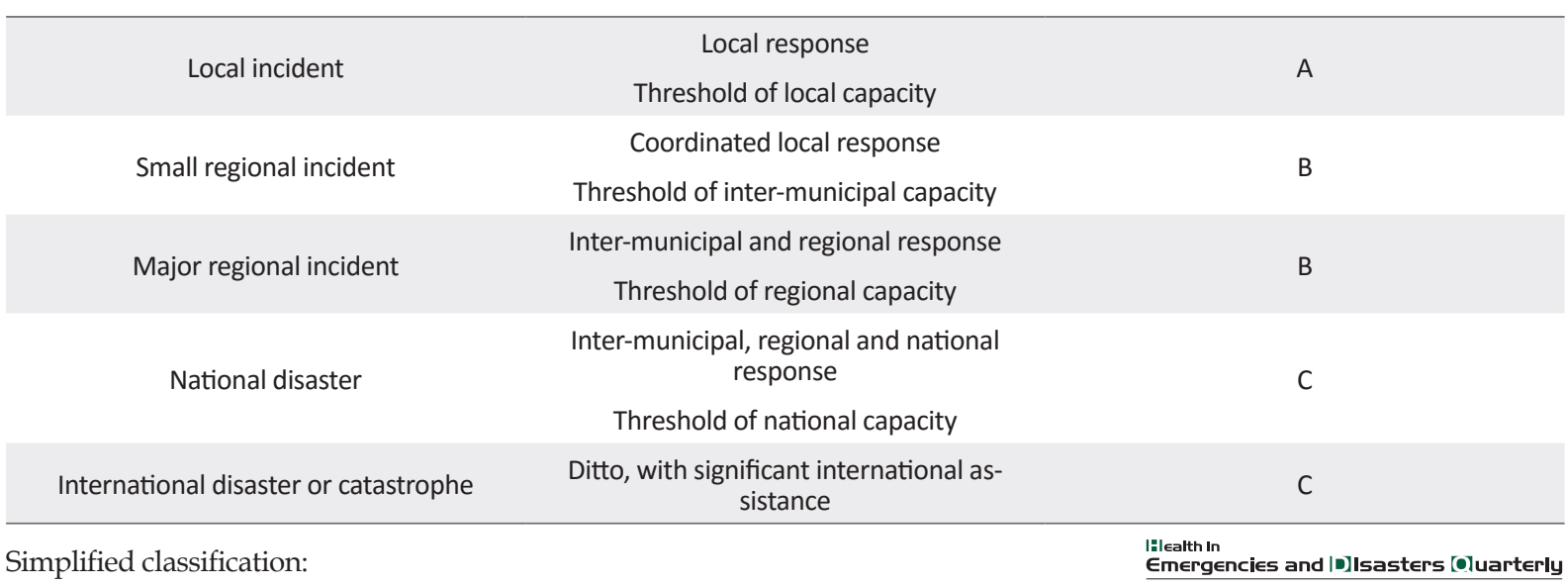

A) Local emergency, B) Regional major incident, C) National (or internationally declared) disaster. 
system is usually somewhat irregular, as it manifests and copes with different speeds and degrees of development.

This, then, is the environment of civilian emergency planning. Generally, the levels of emergency are determined by thresholds of capability (Table 1). When the ability of a municipality to manage a contingency is overwhelmed by the size of the event, it will be necessary to invoke an inter-municipal, regional or national response. Part of the art of emergency planning is to build into the plan the ability to recognize such limitations and trigger a broader response where necessary, by conducting a timely needs assessment when disaster strikes, and by effectively communicating unmet needs to higher levels of authority. In Table 1, the local emergency usually does not require special measures. Emergency planning comes into its own when local resources are too small to cope with the needs generated by a major incident or disaster.

Another aspect of the art of emergency planning is to maintain a balance between creating autonomy and working with other jurisdictions. This is a question of making effective use of the resources that are available in the jurisdiction for which the plan is written, and recognizing the need for reinforcements, hence, interoperability (see below). An emphasis on providing good, robust communications can help create interoperability, and beyond that it will need much discussion, liaison, and joint work between different services. The effort will be repaid by smooth-running operations during an emergency situation.

Lastly, it is important to ensure that planning and the emergency responses it regulates, focused on the beneficiaries, who are the people caught up in the incident or disaster, who either are victims and survivors, or risk becoming such figures unless they are helped and protected. The London Assembly report on the handling of the bombings in London, England, on 7 July 2005, made this point very clearly [2]. The authors of the report felt that the emergency services were too caught up in their own needs and insufficiently focused on those of the victims and survivors. They provided much evidence to support this contention. Emergency response is practised for the general public and its various constituent groups, not for the emergency services. This may seem obvious, but there may be a tendency to overlook it and focus on the needs of the services to the exclusion of the needs of those they serve.

\section{About the Author}

David Alexander is Professor of Risk and Disaster Reduction at University College London. He is also Visiting Professor at the Universities of Bournemouth and
Northumbria (UK), Affiliated Professor at the University of Lund (Sweden) and Research Fellow at the Global Risk Forum GRF Davos (Switzerland). Professor Alexander is Vice-President of the Institute of Civil Protection and Emergency Management and Editor-in-Chief of the International Journal of Disaster Risk Reduction.

\section{References}

[1] United Nations Office for the Co-ordination of Humanitarian Relief. Guidelines on the use of foreign military and civil defence assets in disaster relief: 'Oslo guidelines'. Geneva: United Nations Office for the Co-ordination of Humanitarian Relief; 2007, p. 32.

[2] Greater London Assembly. Report of the 7 July Review Committee. London: Greater London Assembly; 2006, p. 151. 